\title{
An Improved Selective Mapping Method for PAPR Reduction in OFDM/OQAM System
}

\author{
Guobing Cheng, Huilei Li, Binhong Dong, Shaoqian Li \\ National Key Laboratory of Science and Technology on Communications, \\ University of Electronic Science and Technology of China, Chengdu, China \\ Email: guobingcheng12@163.com
}

Received May, 2013

\begin{abstract}
Orthogonal frequency division multiplex/offset QAM (OFDM/OQAM) has been proven to be a promising multi-carrier modulation (MCM) technique for the transmission of signals over multipath fading channels. However, OFDM/OQAM has also the intrinsic disadvantage of high peak-to-average-power ratio (PAPR) that should be alleviated. In this paper, a novel selective mapping (SLM) method is proposed for OFDM/OQAM system. Since the pulse shape may cover a few OFDM symbols, the basic principle of the proposed method is to apply the SLM method in the range of the most relevant OFDM symbols. Analysis and simulation results show that, compared to the existing SLM algorithms for OFDM/OQAM system, the proposed method has better PAPR performance and lower computation complexity.
\end{abstract}

Keywords: OFDM/OQAM; PAPR Reduction; Selective Mapping

\section{Introduction}

Orthogonal frequency division multiplexing (OFDM) is an efficient and popular multi-carrier modulation (MCM) scheme that it has been some practical applications such as the 3rd generation mobile communication system, digital subscriber lines (xDSL) and digital video broadcasting (DVB). However, OFDM has some intrinsic drawbacks. Firstly, the rectangular pulse shape leads to high sensitivity to the inter-carrier interference (ICI). Secondly, its robustness to multi-path propagation effects comes from the insertion of a cyclic prefix (CP), i.e., obtained at the price of a reduced spectral efficiency. Furthermore, it has high out-of-band radiations, leading to severe adjacent channel interference. In order to alleviate these drawbacks, OFDM/offset QAM (OFDM/ OQAM) is another MCM scheme which may be the appropriate alternative [1-3]. Compared to traditional CP-OFDM, OFDM/OQAM may provide a higher useful bit rate since it operates without CP. The introduced pulse shapes with good time-frequency localization (TFL) characteristic lead to more immunity to inter-symbol interference (ISI), ICI and out-of-band radiation [4]. Therefore, it is an efficient transmission technique and has attracted increasing attention.

However, as well as the other kinds of MCM systems, since the resulting OFDM/OQAM signal is the summation over all the statistically independent subcarriers, it has the intrinsic characteristic of high peak-to-ratio (PAPR). For a given power amplifier, it always has a certain linear amplification range and distortion will be created when working at the nonlinear range. Furthermore, the power amplification of signals having a large dynamic range may introduce inter-modulation between subcarriers and cause interferences [5]. These distortion and interference lead to performance degradations, which are in close relation with the PAPR of the signal.

There have been some literatures attributed to the reduction of PAPR in OFDM/OQAM system. In [6], the authors derived an approximate expression of the wellknown complementary cumulative density function (CCDF) for OFDM/OQAM system. It concluded that the expression of CCDF of OFDM/OQAM is similar to that of the OFDM system and the common orthogonal pulse shapes also can provide optimal CCDF performance. In [7], the authors analyzed the application of the partial transmit sequence (PTS) method to OFDM/OQAM system and a novel algorithm based on dynamic programming (DP) joint optimization has been presented to reduced the PAPR. Corresponding to the selective mapping (SLM) method in OFDM system [8], the authors in [9] proposed an overlapped selective mapping (OSLM) method. It shows that the performance increases with the number of SLM codes, but is also dependent on the length of the pulse shape that the longer the length of the pulse shape is, the worse the CCDF performance will be.

In this paper, we also focus on the application of SLM method to OFDM/OQAM system. In contrast to the OSLM method that the selective mapping method is used 
for the real-valued data and all the overlapped OFDM symbols are considered, the proposed method applies the SLM method for the combined complex-valued data and considers the overlap only in the most relevant symbols. The simulation results and analysis show that the proposed method outperforms the OSLM method and it can achieve almost the performance of the SLM algorithm in OFDM system. Furthermore, the calculation complexity is largely reduced. The rest of this paper is organized as follows. In section II, we recall the basics of OFDM/ OQAM system and the OSLM algorithm is presented in section III. We present our improved SLM method for OFDM/OQAM and compare it with OSLM algorithm in section IV. The simulation results and analysis are shown in section V. Brief conclusion is given in section VI.

\section{OFDM/OQAM System Mode}

The baseband version of a continuous-time OFDM/ OQAM transmitting signal can be written as [1]

$$
s(t)=\sum_{n=-\infty}^{+\infty} \sum_{m=0}^{M-1} a_{m, n} e^{j \phi_{m, n}} e^{j 2 \pi m v_{0} t} g\left(t-n \tau_{0}\right),
$$

with $M$ an even number of sub-carriers, $a_{m, n}$ the realvalued symbol conveyed by the sub-carrier of index $m$ during the symbol time of index $n, g$ the pulse shape, $v_{0}$ the subcarrier spacing and $\tau_{0}$ the time offset between the adjacent real part and imaginary part of an OFDM/ OQAM symbol. $v_{0}=1 / T_{0}=1 / 2 \tau_{0}$, with $T_{0}$, the duration of the complex-valued symbols. $\phi_{m, n}$ is an additional phase term given by

$$
\phi_{m, n}=\phi_{0}+\frac{\pi}{2}(m+n)(\bmod \pi),
$$

where $\phi_{0}$ can be arbitrarily chosen.

For a distortion-free channel, perfect reconstruction of real symbols is obtained owing to the following real orthogonal condition

$$
\mathfrak{R}\left\{\left\langle g_{m, n} \mid g_{p, q}\right\rangle\right\}=\mathfrak{R}\left\{\int g_{m, n}(t) g_{p, q}^{*}(t)\right\}=\delta_{m, p} \delta_{n, q}
$$

where $\mathfrak{R}\{\bullet\}$ is the real part operator. $\delta_{m, p}=1$ if $m=p$ and $\delta_{m, p}=0$ if $m \neq p$.

It can be seen from equation (1) that the transmitted signal $s(t)$ is the summation over all the statistically independent subcarriers, if the number of subcarriers $M$ is large enough, the amplitude of $s(t)$ also varies in a large range.

\section{The OSLM METHOD for OFDM/OQAM 3.1. Basic Definition of PAPR in OFDM/OQAM System}

The PAPR is an important parameter to measure the sensitivity to non-linear amplification of transmission schemes having a non-constant envelope [9]. And the
PAPR of the OFDM signals with $M$ carriers in discretetime version is defined as follows [8]:

$$
\operatorname{PAPR}(d B)=10 \log _{10} \frac{\max _{k \in\{0, \ldots, M-1\}}\left\{\left|s_{k}\right|^{2}\right\}}{E\left\{\left|s_{k}\right|^{2}\right\}},
$$

where $s_{k}$ is the OFDM signal and $E\{*\}$ is the mean of $\{*\}$.

Since both OFDM and OFDM/OQAM systems transmit the equivalent of one complex symbol at rate $1 / T_{0}$, even the length of the OFDM/OQAM pulse shape $g$ may be longer than $T_{0}$, it is reasonable to use equation (4) for PAPR measurement in OFDM/OQAM system [9].

The PAPR is a random variable of OFDM/OQAM system and its behavior is to compute the probability to exceed a given threshold $\gamma(d B)$ and the CCDF gives this probability for every $\gamma$, which is given by

$$
\mathrm{CCDF}=\mathrm{P}\{\mathrm{PAPR}>\gamma\}
$$

\subsection{The SLM Method for OFDM System}

The SLM technique is a simple and undistorted processing way to reduce the PAPR of OFDM signals [8]. The basic principle of SLM is to generate different versions of the same OFDM symbol and transmit the one with the lowest value of PAPR, see Figure 1. To create these different versions of the same OFDM symbol, we consider $U$ codes of length $M$ and these codes are such that the initial constellation remains unchanged. If

$c_{m}(m \in 0, \ldots, M-1)$

is a point of a $2^{2 K}-\mathrm{QAM}$ constellation, the randomly generated codes $d_{m}^{(u)}(m, u) \in\{0, \ldots, M-1\} \times\{1, \ldots, U\}$ are such that $d_{m}^{(u)} c_{m}$ is a point of the same $2^{2 K}-$ QAM constellation. In order to retrieve the original data, the receiver requires a perfect knowledge of the used code. Therefore $\log _{2} U$ bits are needed to be transmitted as side information to recover them perfectly, leading to reduction of the useful data rate.

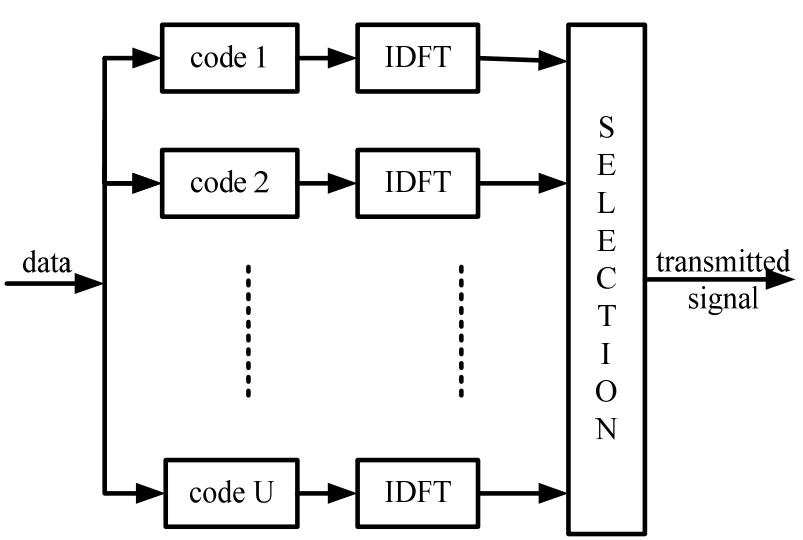

Figure 1. The SLM scheme for PAPR reduction in OFDM system. 


\subsection{The OSLM Method for OFDM/OQAM System}

The SLM for OFDM/OQAM system has a similar structure of SLM for OFDM system. The transmitted data is selected by the lowest PAPR of $\mathrm{U}$ dependent coded symbols. But for the reason that the pulse shape is introduced in OFDM/OQAM system and the length of pulse shape may be longer than the symbol period $T_{0}$, the traditional SLM scheme can not directly be applied to OFDM/OQAM system any more. In order to apply the SLM technique and keep and perfect recovery, the OSLM method is proposed in [9].

Assume the pulse shape is of length $b T_{0}(b \in N)$, then the overlapping is caused by the $(2 b-1)$ pulse shapes, and all the associated overlaps have to be taken into account. The brief description of OSLM is given as follows [9]:

1) Generating $U$ codes of length $M$ and the original symbol $a_{m, n}$ is coded by the code $d_{m}^{(i)}$ and we get the code denoted by $a_{m, n}^{(i)}$.

2) Constructing the $b$ first OFDM symbols. For a pulse shape of length $b T_{0}$, overlaps only occur for duration equal to . Therefore, the first $2 b$ symbols can be randomly chosen. Let $a_{m, n}=a_{m, n}^{(1)}, n \leq 2 b$, then the selected codes and the associated coded symbols are stored as:

$$
A_{2 b}=\left(a_{m, n}^{(1)}\right)_{0 \leq m \leq M-1,1 \leq n \leq 2 b}
$$

3) Considering the $I_{2 b}=\left[2 b \tau_{0},(2 b+1) \tau_{0}\right]$ interval, then the corresponding matrices $A_{2 b+1}^{(i)}$ can be expressed as:

$$
A_{2 b+1}^{(i)}=\left[A_{2 b}\left(a_{m, 2 b+1}^{(i)}\right)_{0 \leq m \leq M-1}\right]
$$

The associated OFDM/OQAM signals are generated for all $i$. Then, to take into account all the modifications brought by the addition of a new pulse shape, the index code $i_{2 b+1}$ selected is the one that provides the lowest PAPR value on the last interval of length $b T_{0}$ and then set $A_{2 b+1}=A_{2 b+1}^{\left(i_{2 b+1}\right)}$.

4) Generalization on the $I_{k}, k>2 b$ interval. Let

$$
A_{k+1}^{(i)}=\left[A_{k}\left(a_{m, k+1}^{(i)}\right)_{0 \leq m \leq M-1}\right],
$$

and proceed as in step 3. Then for a given $i, A_{k+1}^{(i)}$ is a $M \times(k+1)$ matrix. But knowing that any new added pulse shape only corrupts the signal on a $b T_{0}$ length interval, the matrices associated to the different signals can be restricted in time leading to a $M \times 2 b$ matrix $B_{k+1}^{(i)}$ such that:

$$
\left(B_{k+1}^{(i)}\right)_{m, n}=\left(A_{k+1}^{(i)}\right)_{n \in\{k-2 b+1, \ldots, k+1\}}^{m \in\{0, \ldots, \ldots-1\}},
$$

We can see that, for the OSLM method, the selective mapping procedure is carried out in each real symbol period $\tau_{0}$. Therefore, compared to the SLM in each complex symbol period $T_{0}$, it costs twice the calculation quantity while achieves higher PAPR results. On the other hand, the OSLM does not consider the fact that, for the common pulse shapes introduced in OFDM/OQAM system such as the isotropic orthogonal transform algorithm (IOTA) and square-root raised cosine (SRRC), most of the energy is located in the main lobe.

\section{The Proposed SLM for OFDM/OQAM}

In this paper, an improved OSLM algorithm is proposed to reduce the PAPR in OFDM/OQAM system that the data matrix is limited to two OFDM symbols and the SLM algorithm is carried out in each complex-valued OFDM symbol $T_{0}$. The proposed method is depicted as follows.

1) Generating $U$ codes of length $M$ and the original symbol $a_{m, n}$ is coded by the code $d_{m}^{(i)}$ and we get the code denoted by $a_{m, n}^{(i)}$.

2) Constructing the first two OFDM symbols with randomly selection and associated coded symbols are stored as:

$$
A_{2}=\left(a_{m, n}^{(1)}\right)_{0 \leq m \leq M-1,1 \leq n \leq 2}
$$

3) Considering the $I_{3}=\left[2 \tau_{0}, 4 \tau_{0}\right]$ interval, then the corresponding matrices $A_{2 b+1}^{(i)}$ can be expressed as:

$$
A_{3}^{(i)}=\left[\begin{array}{ll}
A_{2} & \left(a_{m, 3}^{(i)}\right)_{0 \leq m \leq M-1}
\end{array}\right]
$$

The associated OFDM/OQAM signals are generated for all $i$. Then the index code $i_{3}$ selected is the one that provides the lowest PAPR value and then set $A_{3}=A_{3}^{\left(i_{3}\right)}$.

4) Generalization on the $I_{k}, k>2$ interval. Let

$$
A_{k+1}^{(i)}=\left[A_{k}\left(a_{m, k+1}^{(i)}\right)_{0 \leq m \leq M-1}\right],
$$

and proceed as in step 3 . Then the index that provides the lowest PAPR value can be selected step by step.

\section{Simulation Results}

In this section, it aims to compare the performance of proposed SLM with OSLM for OFDM/OQAM system and the traditional SLM for OFDM system is also given. The pulse shape introduced in the simulation is the IOTA filter.

Figure 2 and Figure 3 show the results of CCDF to the threshold $\gamma$ and the number of subcarriers are $\mathrm{N}=$ 64 and $\mathrm{N}=128$ respectively. It can be seen that in both scenarios, the proposed method outperforms the OSLM and has almost the same performance to the SLM in OFDM system.

In Figure 4, it shows the CCDF performance of the proposed method in condition of different length of pulse 


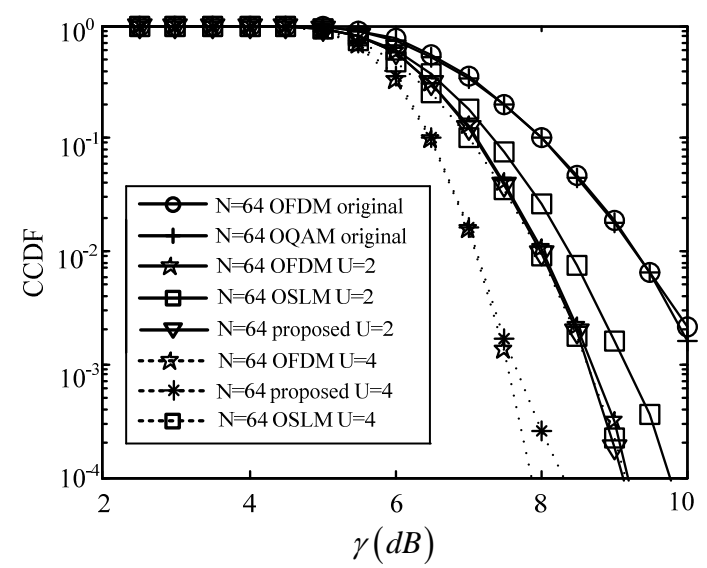

Figure 2. CCDF performance comparison between OFDM, OSLM and the proposed method with the number of subcarriers $N=64$.

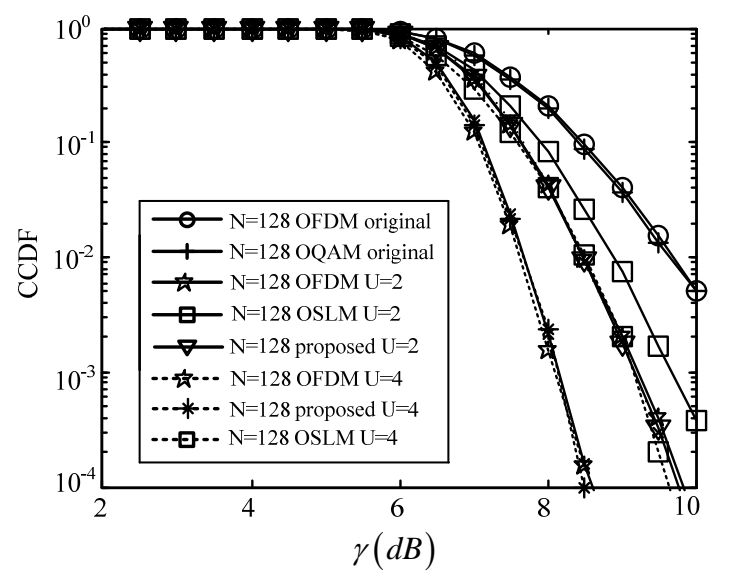

Figure 3. CCDF performance comparison between OFDM, OSLM and the proposed method with the number of subcarriers $\mathbf{N}=128$.

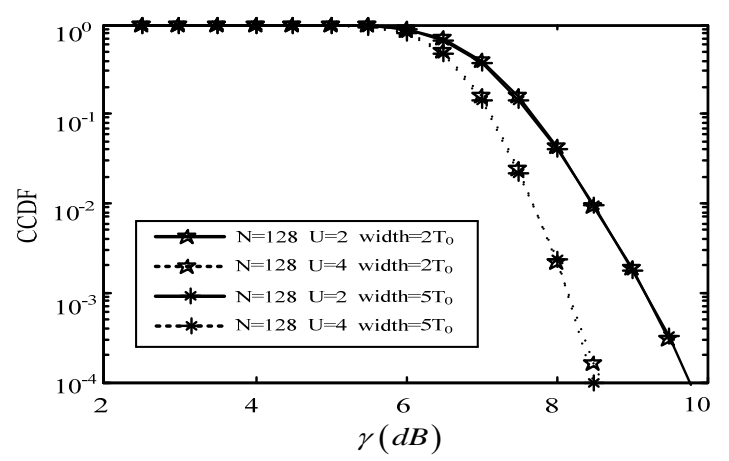

Figure 4. CCDF performance of the proposed method in condition of different length of pulse shape is considered.

shape is considered. We set the calculated length of the relevant symbol to $W=2 T_{0}$ and $W=5 T_{0}$. The simulation results show that they can achieve the similar performance. This indicates that we can limit the calculation matrix to $M \times 2$, leading to that the amount of calcula- tion is greatly reduced.

\section{Conclusions}

In this paper, an improved OSLM method is proposed for OFDM/OQAM system and it achieves a better performance than the OSLM method while with lower calculation complexity. It can be concluded that, as to the SLM method, the OFDM/OQAM system has the similar CCDF performance to that of the OFDM system and has little to do with the length of the pulse shapes.

\section{Acknowledgements}

This work is supported in part by National Sci. \& Tech. Major Project of China under Grant2010ZX03006-00202 and Program for New Century Excellent Talents in University of China ((NCET110058).

\section{REFERENCES}

[1] B. Le Floch, M. Alard and C. Berrou, "Coded Orthogonal Frequency Division Multiplex,” Proceedings of the IEEE, Vol. 83, 1995, pp. 982-996. doi:10.1109/5.387096

[2] H. Bölcskei, "Orthogonal Frequency Division Multiplexing Based on Offset QAM,” in Advances in Gabor analysis. Birkhäuser, 2003.

[3] P. Siohan, C. Siclet and N. Lacaille, "Analysis and Design of OFDM/OQAM Systems Based on Filterbank Theory," IEEE Transactions on Signal Processing, Vol. 50, No. 5, 2002, pp. 1170-1183. doi:10.1109/78.995073

[4] J. F. Du and S. Signell, "Time Frequency Localization of Pulse Shaping Filters in OFD/OQAM Systems,” 6th International Conference on Information, Communications \& Signal Processing, Dec. 2007.

[5] L. Hanzo, M. Miinster, B. J. Choi and T. Keller, "OFDM and MC-CDMA for Broadband Multi-Users Communications," WLANs and Broadcasting, IEEE press. Wiley Series, 2003.

[6] A. Skrzypczak, P. Siohan, and J. P. Javaudin, "Analysis of the Peak-to-average Power Ratio for OFDM/OQAM," IEEE 7th Workshop on Signal Processing Advances in Wireless Communications, July 2006.

[7] D. M. Qu, S. X. Lu and T. Jiang, "Multi-Block Joint Optimization for the Peak-to-average Power Ratio Reduction of FBMC-OQAM Signals,” IEEE Transactions on Signal Processing, Vol. 61, No. 7, 2013, pp. 1605-1613. doi:10.1109/TSP.2013.2239991

[8] R. W. Bauml, R. F. H. Fischer and J. B. Huber, "Reducing the Peak-to-Average Power Ratio of Multicarrier Modulation by Selected Mapping,” Electronics Letters, Vol. 32, No. 22, 1996, pp. 2056-2057. doi:10.1049/el:19961384

[9] A. Skrzypczak, P. Siohan, and J. P. Javaudin, "Reduction of the Peak-to-average Power Ratio for OFDM-OQAM Modulation,” in Proc. 63rd IEEE VTC, May 2006, pp. 2018-2022. 